\title{
APPLICATIONS OF NILMANIFOLD THEORY TO DIOPHANTINE APPROXIMATIONS
}

\author{
JONATHAN BREZIN ${ }^{1}$
}

\begin{abstract}
We indicate one method for applying harmonic analysis of nilmanifolds to problems of uniform distribution mod 1 in $\boldsymbol{R}$.
\end{abstract}

1. Introduction. We are going to illustrate a technique for lising the results of [1] and [3] to prove theorems on the uniform distribution of sequences mod 1 in $\boldsymbol{R}$. Two results will be proved: one classical, the other new. The classical result is $H$. Weyl's theorem (see [5]) that whenever $P$ is a polynomial over $\boldsymbol{R}$ at least one of whose coefficients (other than the constant term) is irrational, the sequence $P(1), P(2), \cdots$ is uniformly distributed mod 1. Our proof of Weyl's theorem is closely related to a proof given by F. Hahn in [4]. Hahn was dealing with affine maps from a torus to itself, and for that reason his methods do not extend in an obvious way to get our new result, which is that whenever $\tau$ is an irrational number and $n$ is an integer greater than 1 , the sequence ${ }^{2} a_{r}=$ $(\tau n !)^{-1}\left(\{\tau r\}^{n}+(-1)^{n+1}[\tau r]^{n}\right)$ is uniformly distributed mod 1. Our proof of this result uses in an essential way certain affine maps of a nilmanifold which is visibly not a torus.

Our computations all deal with functions on a certain nilmanifold to whose description we now turn:

2. The nilmanifold. Let $X_{1}, \cdots, X_{n}$ denote the standard basis for $\boldsymbol{R}^{n}$, and define the linear map $T: \boldsymbol{R}^{n} \rightarrow \boldsymbol{R}^{n}$ by setting $T X_{i}=X_{i+1}$, for $1 \leqq i<n$, and $T X_{n}=0$. We define a semidirect product $\boldsymbol{R}^{n} \cdot \boldsymbol{R}$ by setting

$$
(y, t)\left(y^{\prime}, t^{\prime}\right)=\left(y+\exp (t T) y^{\prime}, t+t^{\prime}\right)
$$

for all $y, y^{\prime} \in \boldsymbol{R}^{n}$ and all $t, t^{\prime} \in \boldsymbol{R}$. (By $\exp (t T)$ we mean $\sum_{j=0}^{n-1}(j !)^{-1} t^{j} T^{j}$.) The group operation for $\boldsymbol{R}^{n} \cdot \boldsymbol{R}$ described in (2.1) is metabelian nilpotent. (This group also appears in [2, Chapter VIII].)

Received by the editors August 4, 1971.

AMS 1969 subject classifications. Primary 2265.

Key words and phrases. Nilmanifold, uniform distribution, diophantine approximation.

${ }^{1}$ Research partially supported by a grant from the National Science Foundation.

2 In what follows, ['] means "integer part" and $\{\cdot\}$ means fractional pari.

(c) American Mathematical Society 1972 
Let $T$ denote the circle group, and let $\chi: R^{n} \rightarrow T$ be a character of $R^{n}$. The character $\chi$ induces a unitary representation of $R^{n} \cdot R$ on $L^{2}(R)$, as follows:

$$
\left(U_{(y, t)}^{x} f\right)(u)=\chi(\exp (u T) y) f(u+t)
$$

for all $(y, t) \in \boldsymbol{R}^{n} \cdot \boldsymbol{R}$ and all $f \in L^{2}(\boldsymbol{R})$. Let $\langle\cdot, \cdot\rangle$ denote the usual inner product in $\boldsymbol{R}^{n}$; then there is a unique element $m \in \boldsymbol{R}^{n}$ such that $\chi(y)=$ $\exp 2 \pi i\langle m, y\rangle$. We will usually write $U^{m}$ in place of $U^{x}$.

The subset $\left\{(m, n ! r): m \in Z^{n}, r \in Z\right\}$ of $\boldsymbol{R}^{n} \cdot \boldsymbol{R}$ is a subgroup of $\boldsymbol{R}^{n} \cdot \boldsymbol{R}$, denoted $\Gamma$. The quotient $\boldsymbol{M}=\left(\boldsymbol{R}^{n} \cdot \boldsymbol{R}\right) / \Gamma$ is a compact nilmanifold. (Note: We use cosets of the form $\Gamma x$.) There is a unique probability measure $\nu$ on $\boldsymbol{M}$ that is invariant under translation by elements of $\boldsymbol{R}^{n} \cdot \boldsymbol{R}$. For each $m \in Z^{n}$, we define (cf. $[3, \S 3]$ ) the "homogenizing" map $\Theta^{m}: L^{2}(R) \rightarrow L^{2}(M, v)$ by

$$
\left(\Theta^{m} f\right)(y, t)=\sum_{k=-\infty}^{\infty}(\exp 2 \pi i\langle m, \exp (n ! k T) y\rangle) f(t+n ! k) .
$$

The properties of $\Theta^{m}$ are described in [3], all we shall need is the obvious fact that if $m \neq 0$, then $\int_{M} \Theta^{m} f d v=0$.

Given an $(n+1)$-tuple $Y=\left(\lambda_{1}, \cdots, \lambda_{n}, \tau\right)$ of real numbers, we define a one-parameter subgroup of $\boldsymbol{R}^{n} \cdot \boldsymbol{R}, \exp (r Y), r \in \boldsymbol{R}$, by setting

$$
y_{j}^{(r)}=\sum_{k=1}^{j} r^{k} \tau^{k-1} \lambda_{j-k+1} / k ! \quad \text { and } \exp (r Y)=\left(y_{1}^{(r)}, \cdots, y_{n}^{(r)}, r \tau\right) .
$$

The main result of $\S 5$ of [1] says that the sequence $\Gamma \exp (Y), \Gamma \exp (2 Y), \cdots$ is uniformly distributed in $\boldsymbol{M}$, with respect to the measure $\nu$, if, and only if, the real numbers $\tau$ and $\lambda_{1}$ are linearly independent over the rational numbers.

3. Weyl's theorem. We are now ready to prove the theorem of Weyl stated in the introduction. It is a routine computation to prove that in order that Weyl's theorem be true, it is sufficient that whenever $P$ is a polynomial over $\boldsymbol{R}$ whose leading coefficient is irrational, the sequence $P(1), P(2), \cdots$ is uniformly distributed $\bmod 1$. Now Weyl's uniform distribution criterion (see [5]) says that for a sequence $a_{r}$ of real numbers to be uniformly distributed mod 1 , it is necessary and sufficient that for every nonzero integer $m$, we have $\lim _{N \rightarrow \infty} N^{-1} \sum_{j=1}^{N} \exp \left(2 \pi i a_{j} m\right)=0$. Thus, the proof of Weyl's theorem comes down to showing that if $P$ is polynomial over $\boldsymbol{R}$ whose leading coefficient is irrational, then

$$
\lim N^{-1} \sum_{j=1}^{N} \exp (2 \pi i m P(j))=0
$$

for every nonzero $m \in Z$. Now we appeal to nilmanifold theory. 
Consider the one-parameter subgroup $\exp (r Y)$, defined in $\$ 2$, in the special case $\tau=n !, \lambda_{1}, \cdots, \lambda_{n}$ arbitrary. Set $m=(0,0, \cdots, 0, \mu) \in Z^{n}$, and let $f$ be any $C_{0}^{\infty}$ function on $R$ with support in $\left[-\frac{1}{2}, \frac{1}{2}\right]$. Then, for any integer $r$,

Now

$$
\Theta^{m} f(\exp (r Y))=f(0) \exp 2 \pi i\left\langle m, \exp (-n ! r T) y^{(r)}\right\rangle .
$$

$$
\begin{aligned}
\left\langle m, \exp (-n ! r T) y^{(r)}\right\rangle & =\mu \sum_{j=0}^{n-1}(-1)^{j}(n ! r)^{j} y_{n-j}^{(r)} / j ! \\
& =\mu \sum_{j=0}^{n-1}(-1)^{j}(j !)^{-1}(n ! r)^{j} \sum_{i=1}^{n-j}(n ! r)^{j}(n ! i !)^{-1} \lambda_{n-j-i+1} \\
& =\mu \sum_{k=1}^{n} \lambda_{n-k+1}(n ! r)^{k}(n ! k !)^{-1} \sum_{j=0}^{k-1}(-1)^{j} k !(j !(k-j) !)^{-1} \\
& =\mu \sum_{k=1}^{n}(-1)^{k+1}(n !)^{k-1}(k !)^{-1} \lambda_{n-k+1} r^{k} .
\end{aligned}
$$

Set $a_{k}=(-1)^{k+1}(n !)^{k-1}(k !)^{-1} \lambda_{n-k+1}$, and define the polynomial $Q$ over $\boldsymbol{R}$ by setting $Q(X)=a_{1} X+\cdots+a_{n} X^{n}$. Then what we have shown is that $\Theta^{m} f(\exp (r Y))=f(0) \exp (2 \pi i \mu Q(r))$. Assume that $\lambda_{1}$ (or equivalently, $a_{n}$ ) is irrational; then the sequence $\exp (Y), \exp (2 Y), \cdots$ is uniformly distributed in $M$, and therefore

$$
\lim _{N \rightarrow \infty} N^{-1} f(0) \sum_{r=1}^{N} \exp (2 \pi i \mu Q(r))=\int_{M} \Theta^{m} f d \nu=0 .
$$

It follows that the sequence $Q(1), Q(2), \cdots$ is uniformly distributed $\bmod 1$. Since $\lambda_{1}, \cdots, \lambda_{n}$ were arbitrary, except for the condition that $\lambda_{1}$ be irrational, our proof of Weyl's theorem is complete.

4. Powers of fractional parts. We next consider what happens when we choose for $\exp (r Y)$ the subgroup got when $\tau$ is irrational, $\lambda_{1}=1$, and $\lambda_{2}=\lambda_{3}=\cdots=\lambda_{n}=0$. Taking $m=(0,0, \cdots, 0, \mu) \in Z^{n}$ and $f \in C_{0}^{\infty}[0,1]$, we get

$$
\Theta^{m} f(\exp (r Y))=f(\{\tau r\}) \exp 2 \pi i\left\langle m, \exp (-[\tau r] T) y^{(r)}\right\rangle,
$$

where $\{\cdot\}=$ the fractional part, and $[\cdot]=$ the integer part. Now

$$
\begin{aligned}
\left\langle m, \exp (-[\tau r] T) y^{(r)}\right\rangle & =\mu \sum_{j=0}^{n-1}(-1)^{j}(j !)^{-1}[\tau r]^{j} y_{n-j}^{(r)} \\
& =\mu(\tau n !)^{-1} \sum_{j=0}^{n-1} n !(j !(n-j) !)^{-1}[\tau r]^{j}(\tau r)^{j} \\
& =\mu(\tau n !)^{-1}\left(\{\tau r\}^{n}+(-1)^{n+1}[\tau r]^{n}\right) .
\end{aligned}
$$


Because $\exp (r Y), r=1,2, \cdots$, is uniformly distributed in $\boldsymbol{M}$, and because $\int_{M} \Theta^{m} f d \nu=0$, we have that for every $\varepsilon>0$ there is an $N_{\varepsilon, f}>0$ such that whenever $N>N_{\varepsilon, f}$,

$$
\left|N^{-1} \sum_{r=1}^{N} \Theta^{m} f(\exp (r Y))\right|<\varepsilon .
$$

Choose $f \in C_{0}^{\infty}[0,1]$ so that $0 \leqq f \leqq 1$ and so that $f(x)=1$ for all $x \in$ $[\varepsilon / 2,1-\varepsilon / 2]$, and set

$$
S(\varepsilon, N)=\{r \in Z: 0<r \leqq N \text { and }\{\tau r\} \in[0, \varepsilon / 2] \cup[1-\varepsilon / 2,1]\} .
$$

Because the sequence $\tau, 2 \tau, 3 \tau, \cdots$ is uniformly distributed mod 1 , there is an $N_{\varepsilon}^{\prime}>0$ such that whenever $N>M_{\varepsilon}^{\prime}$, the number of elements in $S(\varepsilon, N)$ is at most $2 \varepsilon N$.

Consider now the average

$$
A_{N}=N^{-1} \sum_{r=1}^{N} \exp \left(2 \pi i \mu(\tau n !)^{-1}\left(\{\tau r\}^{n}+(-1)^{n+1}[\tau r]^{n}\right)\right) .
$$

We wish to prove that if $\mu \neq 0$, then $\lim _{N \rightarrow \infty} A_{N}=0$. Set

$$
A_{N}^{\prime}=N^{-1} \sum_{r \in S(\varepsilon, N)} \exp \left(2 \pi i \mu(\tau n !)^{-1}\left(\{\tau r\}^{n}+(-1)^{n+1}[\tau r]^{n}\right)\right),
$$

and set $A_{N}^{\prime \prime}=A_{N}-A_{N}^{\prime}$. Because for $N$ large enough, $S(\varepsilon, N)$ contains at most $2 \varepsilon N$ elements, we have $\left|A_{N}^{\prime}\right|<2 \varepsilon$ for $N$ large. As for $A_{N}^{\prime \prime}$, our choice $f$ was made so that $A_{N}^{\prime \prime}=N^{-1} \sum_{r \notin S(\varepsilon, N)} \Theta^{m} f(\exp (r Y))$. It follows, therefore, from the inequality (4.1) that for large $N$ we have $\left|A_{N}^{\prime \prime}\right|<3 \varepsilon$. Hence $\left|A_{N}\right|<5 \varepsilon$ for large $N$, and we have $\lim _{N \rightarrow \infty} A_{N}=0$, as desired. We have thus proved:

THEOREM. Let $\tau$ be an irrational number, and let $n$ be an integer greater than 1 ; then the sequence

$$
a_{r}=(\tau n !)^{-1}\left(\{\tau r\}^{n}+(-1)^{n+1}[\tau r]^{n}\right)
$$

is uniformly distributed mod 1.

We remark that the case $n=2$ reduces to the uniform distribution mod 1 of $\tau r^{2}$, which is not surprising in view of the structure of $\boldsymbol{R}^{2} \cdot \boldsymbol{R}$, as a comparison between $\S 3$ and this section will show. The first interesting case is $n=3$, which again is not surprising, as Hahn's "torus" arguments cease to apply in that case.

\section{BIBLIOGRAPHY}

1. L. Auslander and J. Brezin, Uniform distribution in solvmanifolds, Advances in Math. (to appear). 
2. L. Auslander, L. Green and F. Hahn, Flow's on homogeneous spaces, Ann. of Math. Studies, no. 53, Princeton Univ. Press, Princeton, N.J., 1963. MR 29 \#4841.

3. J. Brezin, Function theory on metabelian solvmanifolds, J. Functional Analysis (to appear).

4. F. J. Hahn, On affine transformations of compact abelian groups, Amer. J. Math. 85 (1963), 428-446. MR 27 \#5889.

5. H. Weyl, Über die gleichverteilung von Zahlen mod. I, Math. Ann. 77 (1916), 313-352.

School of Mathematics, University of Minnesota, Minneapolis, Minnesota 55455

Current address: Department of Mathematics, University of California, Berkeley, California 94720 